This item was submitted to Loughborough's Research Repository by the author.

Items in Figshare are protected by copyright, with all rights reserved, unless otherwise indicated.

\title{
Non-aqueous iminium salt mediated catalytic asymmetric epoxidation
}

PLEASE CITE THE PUBLISHED VERSION

PUBLISHER

(C) Elsevier

LICENCE

CC BY-NC-ND 4.0

\section{REPOSITORY RECORD}

Page, Philip C. Bulman, Benjamin R. Buckley, David Barros, A. John Blacker, Harry Heaney, and Brian A. Marples. 2019. "Non-aqueous Iminium Salt Mediated Catalytic Asymmetric Epoxidation". figshare. https://hdl.handle.net/2134/2368. 
This item was submitted to Loughborough's Institutional Repository by the author and is made available under the following Creative Commons Licence conditions.

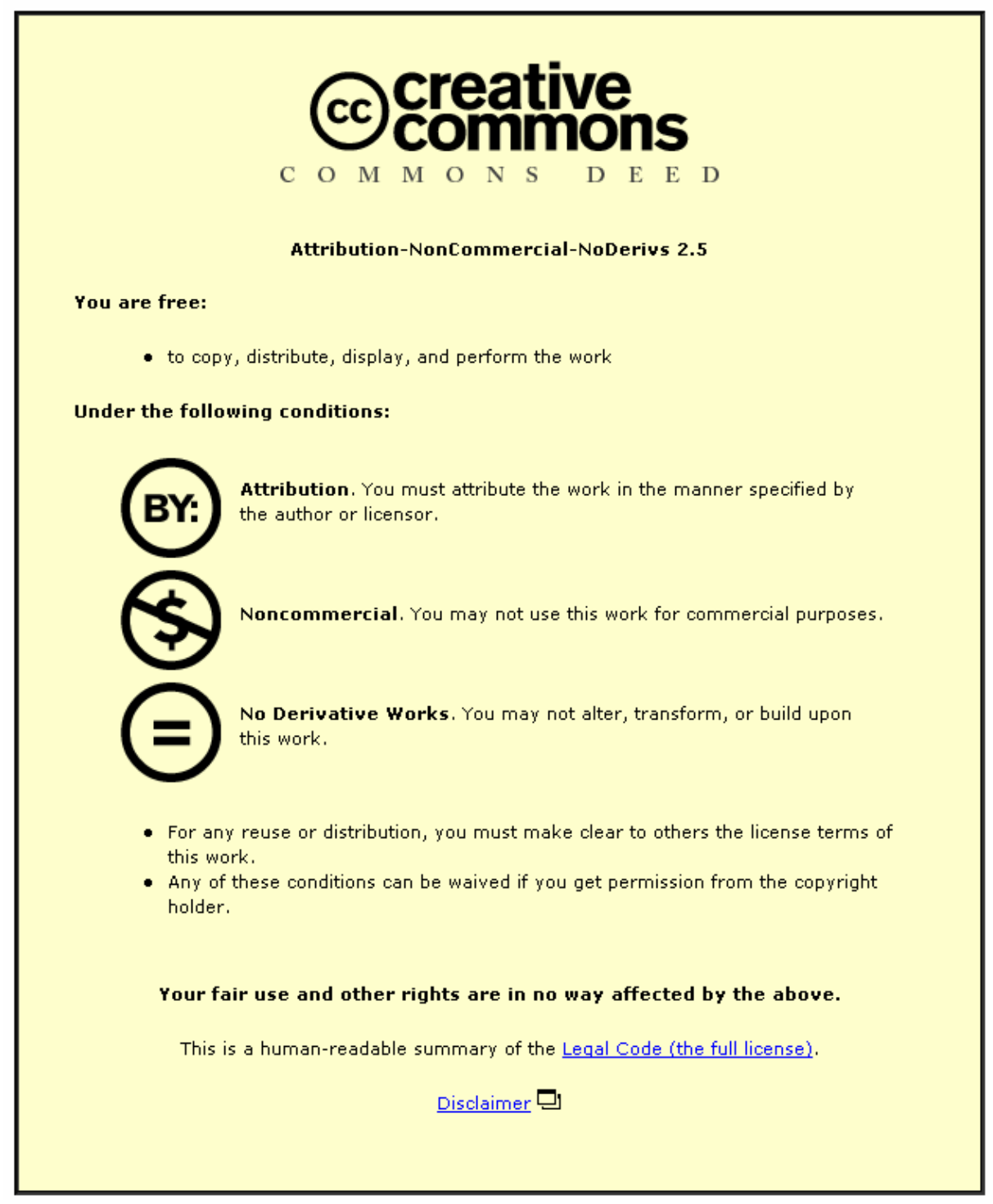

For the full text of this licence, please go to: http://creativecommons.org/licenses/by-nc-nd/2.5/ 
Philip C Bulman Page,* Benjamin R Buckley, David Barros,

A John Blacker, ${ }^{1}$ Harry Heaney, Brian A Marples

Department of Chemistry, Loughborough University,

Loughborough, Leicestershire LE11 3TU, England

p.c.b.page@lboro.ac.uk

Abstract: A range of substituted dihydroisoquinolinium salts has been tested in the catalytic asymmetric epoxidation of simple alkenes using our newly developed non-aqueous conditions employing tetraphenylphosphonium mono-peroxysulfate (TPPP) as oxidant, giving ees of up to $97 \%$.

In 1976 it was established that oxaziridinium salts are extremely reactive reagents for oxygen transfer to nucleophilic substrates. ${ }^{2}$ Since then, there has been significant interest in developing effective iminium salt catalysts for epoxidation of olefins. ${ }^{3}$ The primary oxidant used is oxone, and the reactions are usually performed at $0{ }^{\circ} \mathrm{C}$ in the presence of a base. In addition to an organic solvent, usually acetonitrile, water is an essential cosolvent, required in order to provide sufficient oxone solubility.

We have previously described our approach to new types of cyclic chiral iminium salts, functionalized with chiral units at the nitrogen atom. ${ }^{4}$ These enantiomerically pure iminium salts have been successfully employed in the catalytic asymmetric epoxidation of unfunctionalized alkenes, the dioxane-derived catalysts 1-5 giving ees of up to $c a 60 \%,{ }^{5}$ catalyst 6 giving ees of up to $97 \%,{ }^{6}$ and catalyst 7 ees of up to $95 \%$. $^{7}$<smiles>[R]c1ccc(C2OC(C)(C)OCC2[N+]2=Cc3ccccc3CC2)cc1</smiles>

$1 \mathrm{R}=\mathrm{H} ; 2 \mathrm{R}=\mathrm{NO}_{2}$

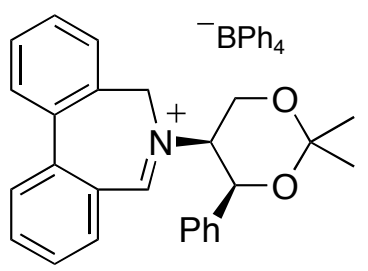

5<smiles>CC1(C)OC[C@@H]([N+]2=Cc3ccccc3CC2)[C@H](c2ccc(S(C)(=O)=O)cc2)O1</smiles>

6

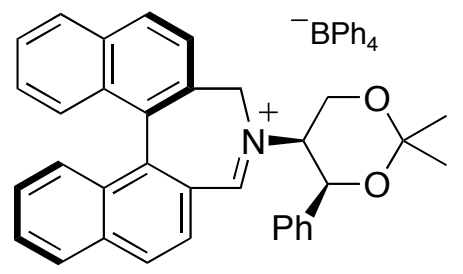

7

\section{$3 \mathrm{R}=\mathrm{SMe} ; 4 \mathrm{R}=\mathrm{OMe}$}

The standard conditions employed by ourselves and others in epoxidation reactions catalysed by iminium salts involve the use of oxone as stoicheiometric oxidant, a base ( $2 \mathrm{~mol}$ eq of $\mathrm{Na}_{2} \mathrm{CO}_{3}$ per eq of oxone) and water / acetonitrile as solvent mixture.

The principal limitation of this system is the restricted range of temperatures at which the epoxidation can be performed ( $\mathrm{ca}-5^{\circ} \mathrm{C}$ to ambient). The upper temperature limit results from the relatively fast decomposition of oxone in basic media at room temperature. The lower limit is determined by the requirement for an aqueous solution (the normal ratio of solvents is $\left(\mathrm{CH}_{3} \mathrm{CN}: \mathrm{H}_{2} \mathrm{O} ; 1: 1\right)$; aqueous acetonitrile freezes at $-8{ }^{\circ} \mathrm{C}$. 
An additional drawback arising from the dependency on oxone is the large quantity of inorganic salt byproducts produced in the reaction.

We have recently described new reaction conditions for iminium salt-catalysed epoxidation, which eliminate the use of both water and base. ${ }^{8}$ This was achieved through the identification of an effective oxidant, tetraphenylphosphonium monoperoxysulfate (TPPP), that is soluble in organic solvents. When oxone is treated with tetraphenylphosphonium chloride, TPPP is produced as a colourless solid (Equation 1). TPPP has previously been used for the oxidation of manganese(III) porphyrins. ${ }^{9}$

$$
\mathrm{Ph}_{4} \mathrm{P}^{+} \mathrm{Cl}^{-}+\text {Oxone }\left(\mathrm{KHSO}_{4}: 2 \mathrm{KHSO}_{5}: \mathrm{K}_{2} \mathrm{SO}_{4}\right) \rightarrow \mathrm{Ph}_{4} \mathrm{P}^{+}\left(\mathrm{HSO}_{5}\right)^{-}
$$

Equation 1

We were pleased to find that the use of TPPP in place of oxone induces efficient epoxidation in organic solvents in the absence of water, and furthermore that the presence of base is now not required; indeed, addition of base actually suppresses the epoxidation process. Use of this oxidant also enables greater scope for reaction monitoring and analysis, as water is no longer required. Reactions can be carried out at low temperature, and, when carried out in deuteriated solvent, monitored by NMR spectroscopy. We describe herein the catalytic asymmetric epoxidation of several unfunctionalized alkenes using catalysts 2-6 under the new anhydrous conditions, employing (TPPP) as stoichieometric oxidant. The catalysts were first tested in the asymmetric epoxidation of 1-phenycyclohexene at varying temperatures using TPPP in dichloromethane (Table 1).

As the temperature is reduced there is an increase in enantiomeric excess at the expense of rate of conversion to epoxide. It is interesting that, while catalyst 1 gives the $(-)-(1 S, 2 S)$ epoxide, when the catalyst contains a strongly electron-withdrawing nitro or sulfone substituent (catalysts $\mathbf{2}$ and $\mathbf{6}$ ), a change in the configuration of the enantiomer of epoxide formed preferentially, to the $(+)-(1 R, 2 R)$ isomer, is observed in every case.

The thiomethyl catalyst 3 also gives the $(+)-(1 R, 2 R)$ enantiomer of 1-phenycyclohexene preferentially, but only between temperatures of $0{ }^{\circ} \mathrm{C}$ and $-50^{\circ} \mathrm{C}$ (Table 1 , entries $3,8,12$ ); at $-78{ }^{\circ} \mathrm{C}$ the $(-)-(1 S, 2 S)$ enantiomer is preferentially formed (Table 1, entry 18). We reason that this is due to rapid oxidation of the sulfide to the sulfone at temperatures above $-78^{\circ} \mathrm{C}$, generating catalyst 6 in situ. Catalyst 4 , which contains an electron-donating methoxy substituent, and has the opposite absolute stereochemistry to catalysts $\mathbf{1}, \mathbf{2}, \mathbf{3}$ and 6, produces the $(+)-(1 R, 2 R)$ epoxide enantiomer preferentially, regardless of temperature. Catalyst 4 thus exhibits the same facial selectivity as unsubstituted catalyst $\mathbf{1}$ (and catalyst $\mathbf{3}$ at low temperatures).

In the case of catalyst 3 , to prove that the active catalyst at temperatures above $-78{ }^{\circ} \mathrm{C}$ was indeed the corresponding sulfone 6, a standard epoxidation reaction was performed in deuteriated dichloromethane solution, and the reaction mixture subjected to ${ }^{1} \mathrm{H}$ NMR spectroscopic analysis. The chemical shift for the methyl group attached to sulfur had shifted from $\delta 2.42$ to 3.03 , confirming that the sulfide had been 
oxidized to the sulfone, by comparison with authentic catalyst 6. The sulfoxide intermediate was not observed.

Table 1: Catalytic asymmetric epoxidation of 1-phenycyclohexene using TPPP in $\mathrm{CH}_{2} \mathrm{Cl}_{2}{ }^{\mathrm{a}}$

\begin{tabular}{|c|c|c|c|c|c|}
\hline Entry & Temp/ $^{\circ} \mathrm{C}$ & Catalyst $^{\mathrm{b}}$ & Conversion/\% $^{\mathrm{c}}$ & ee/\% & Configuration $^{\mathrm{e}}$ \\
\hline 1 & 0 & 1 & 100 & 16 & $(-)-(1 S, 2 S)$ \\
2 & & 2 & 100 & 25 & $(+)-(1 R, 2 R)$ \\
3 & & 3 & 72 & 16 & $(+)-(1 R, 2 R)$ \\
4 & & $4^{\mathrm{f}}$ & - & - & - \\
5 & & 6 & 100 & 33 & $(+)-(1 R, 2 R)$ \\
\hline 6 & -30 & 1 & - & - & - \\
7 & & 2 & 100 & 28 & $(+)-(1 R, 2 R)$ \\
8 & & 3 & 92 & 33 & $(+)-(1 R, 2 R)$ \\
9 & & $4^{\mathrm{f}}$ & 76 & 25 & $(+)-(1 R, 2 R)$ \\
10 & & 6 & 100 & 36 & $(+)-(1 R, 2 R)$ \\
\hline 11 & -45 & 1 & 70 & $37^{\mathrm{g}}$ & $(-)-(1 S, 2 S)$ \\
12 & & 2 & 54 & 30 & $(+)-(1 R, 2 R)$ \\
13 & & 3 & 67 & 33 & $(+)-(1 R, 2 R)$ \\
14 & & $4^{\mathrm{f}}$ & - & - & - \\
15 & & 6 & 96 & 42 & $(+)-(1 R, 2 R)$ \\
\hline 16 & -78 & 1 & 15 & $23^{\mathrm{h}}$ & $(-)-(1 S, 2 S)$ \\
17 & & 2 & 0 & - & - \\
18 & & 3 & 15 & $50^{\mathrm{h}}$ & $(-)-(1 S, 2 S)$ \\
19 & & $4^{\mathrm{f}}$ & 13 & $29^{\mathrm{h}}$ & $(+)-(1 R, 2 R)$ \\
20 & & 6 & - & - & - \\
\hline
\end{tabular}

${ }^{a}$ Epoxidation conditions: iminium salt (10 mol\%), TPPP (2 eq), $\mathrm{CH}_{2} \mathrm{Cl}_{2}, 4$ h. ${ }^{\text {b }}$ Catalyst configuration $(4 S, 5 S)$ unless otherwise stated. ${ }^{c}$ Conversions were evaluated from the ${ }^{1} \mathrm{H}$ NMR spectra by integration of alkene and epoxide signals. ${ }^{\mathrm{d}}$ Enantiomeric excesses were determined by ${ }^{1} \mathrm{H}$ NMR spectroscopy in the presence of $(+)-\mathrm{Eu}(\mathrm{hfc})_{3}(0.1 \mathrm{~mol} \mathrm{eq}) .{ }^{\mathrm{e}}$ The absolute configurations of the major enantiomers were determined by comparison of optical rotation with literature values. ${ }^{\mathrm{f}}$ Catalyst configuration $(4 R, 5 R) .{ }^{\mathrm{g}}$ Reaction carried out at $-40{ }^{\circ} \mathrm{C}$. ${ }^{\mathrm{h}}$ Reaction time $8 \mathrm{~h}$.

In order to determine the effects of solvent on the reaction, several other solvents were screened using our three most enantioselective catalysts $\mathbf{1}, 4$ and $\mathbf{6}$ (Table 2). TPPP was found to be insoluble in carbon tetrachloride, ethyl acetate and dimethoxyethane. In dimethylformamide, the TPPP dissolved but no reaction occurred. TPPP is also soluble in dichloroethane, however, and epoxidation reactions of 1phenylcyclohexene performed in this solvent gave almost identical results to those obtained with dichloromethane for catalysts 6 and 4. Curiously, catalyst $\mathbf{1}$ was far less reactive in this medium and gave a 
reduced ee (24\% compared to $37 \%)$. When the reactions were repeated in chloroform, we observed a dramatic decrease in ee for catalyst $\mathbf{4}$, while catalyst $\mathbf{1}$ gave similar results to those obtained in dichloromethane (33\% ee). Catalyst 6 in chloroform, however, gave the best ee for 1-phenylcyclohexene (48\% ee) that we have observed with this series of iminium salt catalysts. Interestingly, in acetonitrile at -30 ${ }^{\circ} \mathrm{C}$ we observed a similar degree of enantioselectivity to that seen in the reaction carried out in chloroform (48\% ee, $(+)-(1 R, 2 R)$ enantiomer), but producing the opposite (-)-(1S,2S)-enantiomer of phenycyclohexene oxide in $45 \%$ ee. The origins of these solvent effects upon enantioselectivity remain unclear.

Table 2: Asymmetric epoxidation of 1-phenylcyclohexene using various solvents ${ }^{\text {a }}$

\begin{tabular}{|c|c|c|c|c|c|}
\hline Solvent & Catalyst $^{\mathrm{b}}$ & Time/h & Conversion/\% $^{\mathrm{c}}$ & ee/\% $^{\mathrm{d}}$ & Configuration $^{\mathrm{e}}$ \\
\hline \hline $\mathrm{CH}_{3} \mathrm{CN}$ & $\mathbf{1}$ & 1.0 & 42 & $43^{\mathrm{f}}$ & $(-)-(1 S, 2 S)$ \\
& $\mathbf{4}^{\mathbf{g}}$ & 0.2 & 30 & 44 & $(+)-(1 R, 2 R)$ \\
& $\mathbf{6}^{\mathrm{f}}$ & 2.5 & 89 & 45 & $(-)-(1 S, 2 S)$ \\
\hline $\mathrm{CH}_{2} \mathrm{Cl}_{2}$ & $\mathbf{1}$ & 4 & 70 & $37^{\mathrm{f}}$ & $(-)-(1 S, 2 S)$ \\
& $\mathbf{4}^{\mathbf{g}}$ & 4 & 76 & 25 & $(+)-(1 R, 2 R)$ \\
& $\mathbf{6}$ & 4 & 100 & 36 & $(+)-(1 R, 2 R)$ \\
\hline $\mathrm{C}_{2} \mathrm{H}_{4} \mathrm{Cl}_{2}$ & $\mathbf{1}$ & 24 & 29 & 24 & $(-)-(1 S, 2 S)$ \\
& $\mathbf{4}^{\mathbf{g}}$ & 24 & 87 & 17 & $(+)-(1 R, 2 R)$ \\
& $\mathbf{6}$ & 4 & 97 & 32 & $(+)-(1 R, 2 R)$ \\
\hline $\mathrm{CHCl}_{3}$ & $\mathbf{1}$ & 24 & 52 & 33 & $(-)-(1 S, 2 S)$ \\
& $4^{\mathbf{g}}$ & 24 & 73 & 11 & $(+)-(1 R, 2 R)$ \\
& $\mathbf{6}$ & 12 & 100 & 48 & $(+)-(1 R, 2 R)$ \\
\hline
\end{tabular}

${ }^{a}$ Epoxidation conditions: iminium salt $(10 \mathrm{~mol} \%)$, TPPP $(2 \mathrm{eq})$, solvent, $-30{ }^{\circ} \mathrm{C} .{ }^{\mathrm{x}}$ Catalyst configuration $(4 S, 5 S)$. ${ }^{\text {b }}$ Catalyst configuration $(4 S, 5 S)$ unless otherwise stated. ${ }^{c}$ Conversions were evaluated from the ${ }^{1} \mathrm{H}$ NMR spectra by integration of alkene and epoxide signals. ${ }^{d}$ Enantiomeric excesses were determined by ${ }^{1} \mathrm{H}$ NMR spectroscopy in the presence of $(+)-\mathrm{Eu}(\mathrm{hfc})_{3} \quad\left(0.1 \mathrm{~mol}\right.$ eq). ${ }^{\mathrm{e}}$ The absolute configurations of the major enantiomers were determined by comparison of optical rotation with literature values. ${ }^{\mathrm{f}}$ Reaction carried out at $-40{ }^{\circ} \mathrm{C} .{ }^{\mathrm{g}}$ Catalyst configuration $(4 R, 5 R)$.

Using our most enantioselective catalyst 6, we next carried out asymmetric epoxidations of several other unfunctionalized alkenes in acetonitrile and in choloroform solution at a standard temperature of $-40{ }^{\circ} \mathrm{C}$, using $10 \mathrm{~mol} \%$ catalyst (Table 3). Remarkably, changing the reaction solvent was found to switch the major enantiomer of epoxide generated from all trans- and tri-substituted alkenes tested. Further, trans-stilbene, usually a poor substrate with our catalysts (less than $5 \%$ ee was observed under our original aqueous conditions using the same catalyst and oxone), afforded the $(+)-(R, R)$ enantiomer of trans-stilbene oxide in $67 \%$ ee when the reaction was carried out in chloroform solution, but the $(-)-(S, S)$ isomer, in $30 \%$ ee, when performed in acetonitrile. With the exception of $\alpha$-methylstilbene, it is clear from table 3 that chloroform is 
the solvent of choice for catalyst $\mathbf{6}$, and the enantiomeric excesses are far better than those observed when employing TPPP in acetonitrile as well as in the original aqueous conditions using oxone.

Table 3: Catalytic asymmetric epoxidation of various alkenes using TPPP and catalyst $6^{\text {a }}$

\begin{tabular}{|c|c|c|c|c|c|c|c|c|c|}
\hline Solvent & \multicolumn{3}{|c|}{ Oxone $/ \mathrm{CH}_{3} \mathrm{CN} / \mathrm{H}_{2} \mathrm{O}$} & \multicolumn{3}{|c|}{$\mathrm{CH}_{3} \mathrm{CN}$} & \multicolumn{3}{|c|}{$\mathrm{CHCl}_{3}$} \\
\hline Alkene & $\begin{array}{c}\text { Yield/ } \\
\%^{\mathrm{b}} \\
\end{array}$ & $\begin{array}{l}\text { ee/ } \\
\%^{\mathrm{c}} \\
\end{array}$ & Config $^{\mathrm{d}}$ & $\begin{array}{c}\text { Yield/ } \\
\%^{\mathrm{b}} \\
\end{array}$ & $\begin{array}{l}\text { ee/ } \\
\% \\
\end{array}$ & Config $^{\mathrm{d}}$ & $\begin{array}{c}\text { Yield/ } \\
\%^{\mathrm{b}} \\
\end{array}$ & $\begin{array}{l}\text { ee/ } \\
\%^{c}\end{array}$ & Config $^{\mathrm{d}}$ \\
\hline & $100^{\mathrm{e}}$ & 39 & $(-)-(1 S, 2 S)$ & 73 & 45 & $(-)-(1 S, 2 S)$ & 77 & 48 & $(+)-(1 R, 2 R)$ \\
\hline & $100^{\mathrm{e}}$ & $<5$ & $(-)-(S, S)$ & $13^{\mathrm{e}}$ & 30 & $(-)-(S, S)$ & 31 & 67 & $(+)-(R, R)$ \\
\hline & $100^{\mathrm{e}}$ & 32 & $(-)-(1 S, 2 R)$ & 42 & 48 & $(-)-(1 S, 2 R)$ & 35 & 2 & $(+)-(1 R, 2 S)$ \\
\hline & $100^{\mathrm{e}}$ & 50 & $(+)-(S)$ & 25 & 27 & $(+)-(S)$ & $11^{\mathrm{e}}$ & 63 & $(-)-(R)$ \\
\hline & $100^{\mathrm{e}}$ & 47 & $(-)-(1 S, 2 R)$ & 56 & 38 & $(-)-(1 S, 2 R)$ & 98 & 59 & $(+)-(1 R, 2 S)$ \\
\hline & - & - & - & 71 & 53 & $(+)-(1 S, 2 R)$ & 85 & 70 & $(+)-(1 S, 2 R)$ \\
\hline & - & - & - & - & - & - & 83 & 61 & $(+)-(1 S, 2 R)$ \\
\hline & 61 & 45 & $(-)-(1 S, 2 R)$ & 80 & 56 & $(-)-(1 S, 2 R)$ & 89 & 82 & $(-)-(1 S, 2 R)$ \\
\hline & - & - & - & - & - & - & 52 & 88 & $(-)-(1 S, 2 S)$ \\
\hline & - & - & - & - & - & - & 76 & 93 & $(-)-(1 S, 2 S)$ \\
\hline & - & - & - & 63 & 80 & $(-)-(1 S, 2 S)$ & 59 & 97 & $(-)-(1 S, 2 S)$ \\
\hline
\end{tabular}

${ }^{\text {a }}$ Epoxidation conditions: iminium salt $6(10 \mathrm{~mol} \%)$, TPPP $(2 \mathrm{eq}),-40{ }^{\circ} \mathrm{C}, 24 \mathrm{~h} .{ }^{\mathrm{b}}$ Isolated yields unless otherwise stated. ${ }^{c}$ Enantiomeric excesses were determined by ${ }^{1} \mathrm{H}$ NMR spectroscopy in the presence of $(+)-\mathrm{Eu}(\mathrm{hfc})_{3}(0.1 \mathrm{~mol} \mathrm{eq})$ or by chiral HPLC using a Chiracel OD column. ${ }^{\mathrm{d}}$ The absolute configurations of the major enantiomers were determined by comparison of optical rotation with literature values. ${ }^{\mathrm{e}}$ Conversions, evaluated from the ${ }^{1} \mathrm{H}$ NMR spectra by integration of alkene and epoxide signals.

Good enantiomeric excesses were also observed for cis- $\alpha$-methyl styrene (70\% ee) and dihydronaphthalene ( $82 \%$ ee). The level of enantioselectivity observed here is remarkable given that the corresponding reaction carried out under our standard aqueous conditions with oxone afforded dihydronaphthalene oxide in only $45 \%$ ee. The enantiomeric excess for the epoxidation of indene is somewhat lower ( $61 \%$ ee), but is still much higher than other reported ees for this epoxidation when mediated by oxaziridinium salts. ${ }^{5}$ Epoxidation of the non-aryl cis-hept-2-ene produced epoxide with quantitative conversion but with low ee. Electron-deficient alkenes are also poor substrates.

Finally, we turned our attention to the epoxidation of benzopyran substrates. We were delighted to observe excellent enantioselectivities in the epoxidations of 6-nitro-, 6-chloro-, and 6-cyano- 2,2- 
dimethylbenzopyrans, with ees up to an unprecedented $97 \% .^{6}$ It is interesting to note here that, in these cases, whichever solvent was employed, the same enantiomer of epoxide was observed.

\section{Conclusions}

A range of iminium salt asymmetric epoxidation catalysts has been tested under non-aqueous conditions at various temperatures in a number of solvents; from these we have identified catalyst $\mathbf{6}$ as one of the most enantioselective. The reaction solvent has a profound effect on the epoxidation of trans- and tri-substituted alkenes, and in many cases we are able to produce either enantiomer as the major product by choice of acetonitrile or chloroform as solvent. In terms of enantiomeric excesses, the optimum reaction temperature appears to be $-40^{\circ} \mathrm{C}$ and chloroform the solvent of choice. These conditions have provided enantiomeric excesses of up to $97 \%$, in the epoxidation of 6-cyano-2,2-dimethylbenzopyran.

\section{Acknowledgments}

This investigation has enjoyed the support of the EPSRC and Avecia Life Science Molecules. We are also indebted to the EPSRC Mass Spectrometry Unit, Swansea.

\section{Experimental}

General Details. All infrared spectra were obtained using a Perkin-Elmer Paragon 1000 FT-IR spectrophotometer; thin film spectra were acquired using sodium chloride plates. All ${ }^{1} \mathrm{H}$ and ${ }^{13} \mathrm{C}$ NMR spectra were measured at 250.13 and $62.86 \mathrm{MHz}$ with a Bruker AC $250 \mathrm{MHz}$ spectrometer or at 400.13 and 100.62 MHz with a Bruker DPX $400 \mathrm{MHz}$ spectrometer, in deuteriochloroform solution unless otherwise stated, using TMS (tetramethylsilane) as the internal reference. Mass spectra were recorded using a JeolSX102 instrument utilizing electron-impact (EI), fast atom bombardment (FAB), and by the EPSRC national mass spectrometry service at the University of Wales, Swansea, utilising electrospray (ES.). Analysis by GCMS utilized a Fisons GC 8000 series (AS 800), using a 15 m x 0.25 mm DB-5 column and an electron-impact low resolution mass spectrometer. Melting points were recorded using an ElectrothermalIA 9100 melting point instrument and are uncorrected. Optical rotation values were measured with an Optical Activity-polAAar 2001 instrument, operating at $\lambda=589 \mathrm{~nm}$, corresponding to the sodium D line, at the temperatures indicated. Microanalyses were performed on a Perkin Elmer Elemental Analyser 2400 CHN. All chromatographic manipulations used silica gel as the adsorbent. Reactions were monitored using thin layer chromatography (TLC) on aluminium-backed plates coated with Merck Kieselgel 60 F254 silica gel. TLC plates were visualised by UV radiation at a wavelength of $254 \mathrm{~nm}$, or stained by exposure to an ethanolic solution of phosphomolybdic acid (acidified with concentrated sulphuric acid), followed by charring where appropriate. Reactions requiring anhydrous conditions were carried out using glassware dried overnight at $150^{\circ} \mathrm{C}$, under a nitrogen atmosphere unless otherwise stated. Reaction solvents were used as obtained commercially unless otherwise stated. Light petroleum (b.p. $40-60{ }^{\circ} \mathrm{C}$ ) was distilled from calcium chloride prior to use. Ethyl acetate was distilled over calcium sulphate or chloride. Dichloromethane was distilled over calcium hydride. Tetrahydrofuran was distilled under a nitrogen atmosphere from the sodium/benzophenone ketyl radical or from lithium aluminium hydride. Enantiomeric excesses were determined either by proton nuclear magnetic resonance spectroscopy in the presence of europium (III) 
tris[3-(hepta-fluropropylhydroxymethylene)-(+)-camphorate] as the chiral shift reagent, or by chiral HPLC using a Chiracel OD column on a TSP Thermo-Separating-Products Spectra Series P200 instrument, with a TSP Spectra Series UV100 ultra-violet absorption detector set at $254 \mathrm{~nm}$ and a Chromojet integrator.

\section{General procedure for the preparation of dihydroisoquinolinium salts from 2-(2-bromoethyl) benzaldehyde and primary amines.}

2-(2-Bromoethyl)benzaldehyde (1.60 equiv., 1.10 if freshly distilled) was cooled using an ice bath, and a solution of the amine in ethanol $(10 \mathrm{ml}$ per $\mathrm{g}$ of amine) was added dropwise. After the addition was complete, the ice bath was removed and the reaction mixture stoppered to retain $\mathrm{HBr}$, and stirred overnight. A solution of sodium tetraphenylborate or other anion-exchanging salt (1.10 equiv.) in the minimum amount of acetonitrile was added in one portion to the reaction mixture. After stirring for $5 \mathrm{~min}$, the organic solvents were removed under reduced pressure. Ethanol was added to the residue, followed by water. The resulting precipitate was collected by filtration and washed with additional ethanol followed by diethyl ether. If no solid materialized after the addition of the water, the mixture was allowed to settle and the ethanol/water phase was decanted. The gummy residue was triturated in hot ethanol or methanol, inducing precipitation of the organic salt immediately or upon slow cooling of the hot alcoholic solution. Small quantities of acetonitrile may be added during this process to aid solubility.

(+)-N-((4S,5S)-2,2-Dimethyl-4-phenyl-1,3-dioxan-5-yl)-3,4-dihydroisoquinolinium tetraphenylborate (1). 5

Prepared according to the general procedure from $(4 S, 5 S)$-5-amino-2,2-dimethyl-4-phenyl-1,3-dioxane (3.00 $\mathrm{g}, 14.4 \mathrm{mmol})$, and purified by recrystallization from acetone/diethyl ether to give $\mathbf{1}$ as a yellow solid (6.93 g, 75\%), m.p. $169-170{ }^{\circ} \mathrm{C} ;[\alpha]^{20}{ }_{\mathrm{D}}+38.6^{\circ}$ (c 2.70, $\mathrm{CH}_{3} \mathrm{CN}$ ); $v_{\max } / \mathrm{cm}^{-1}$ (nujol) 1637, 1603, 1571, 1480, 1266, 1202, 1166, 1108, 1073; $\delta_{\mathrm{H}}\left(250 \mathrm{MHz} ; \mathrm{CD}_{3} \mathrm{CN}\right), 1.65$ (3 H, s), $1.94(3 \mathrm{H}, \mathrm{s}), 2.39-2.48(1 \mathrm{H}, \mathrm{m}), 2.70-2.82$ (1 H, m), 3.25-3.40 (1 H, m), 3.81-3.97 (1 H, m), 4.00-4.10 (1 H, m), 4.30 (1 H, d, J $13.7 \mathrm{~Hz}), 4.58(1 \mathrm{H}, \mathrm{dd}$, $J$ 13.7, 3.1 Hz), $5.70(1 \mathrm{H}, \mathrm{d}, J 2.8 \mathrm{~Hz}), 6.81(4 \mathrm{H}, \mathrm{t}, J 7.2 \mathrm{~Hz}), 7.35-7.40(6 \mathrm{H}, \mathrm{m}), 7.46(1 \mathrm{H}, \mathrm{t}, J 7.3 \mathrm{~Hz})$, 7.65-7.74 (2 H, m), $8.92(1 \mathrm{H}, \mathrm{s}) ; \delta_{\mathrm{C}}\left(62.50 \mathrm{MHz} ; \mathrm{CD}_{3} \mathrm{CN}\right) 17.9,24.1,28.7,51.6,61.4,65.5,70.7$, 104.9, $121.9,124.3,125.4,125.7,128.1,128.5,128.6,128.0,134.4,135.8,137.0,137.7,138.7,163.5,167.5 ; \mathrm{m} / \mathrm{z}$ $322.1809 ; \mathrm{C}_{21} \mathrm{H}_{24} \mathrm{NO}_{2}$ (cation) requires 322.1807.

\section{(+)-N-((4S,5S)-2,2-Dimethyl-4-(4-nitrophenyl)-1,3-dioxan-5-yl)-3,4-dihydroisoquinolinium}

\section{tetraphenylborate $(2) .^{5}$}

Prepared according to the general procedure from (+)-(4S,5S)-5-amino-2,2-dimethyl-4-(4-nitrophenyl)-1,3dioxane $(0.19 \mathrm{~g}, 0.8 \mathrm{mmol})$, and purified by recrystallization from $\mathrm{CH}_{2} \mathrm{Cl}_{2} /$ hexane to give 2 as yellow plates (0.36 g, 74\%); m.p. $176-178^{\circ} \mathrm{C}$ (dec.); $[\alpha]^{20}{ }_{\mathrm{D}}+107.7^{\circ}$ (c=1.30, acetone); Found C, 77.73; H, 6.23; N, 4.00; $\mathrm{C}_{45} \mathrm{H}_{43} \mathrm{BN}_{2} \mathrm{O}_{4} \cdot 0.5 \mathrm{H}_{2} \mathrm{O}$ requires: $\mathrm{C}, 77.66 ; \mathrm{H}, 6.38 ; \mathrm{N}, 4.03$; $v_{\max }\left(\right.$ film) $/ \mathrm{cm}^{-1} 1635,1604,1571,1524,1478$, 1384, 1202, 1163, 1107, 1032; $\delta_{\mathrm{H}}(400 \mathrm{MHz}$; acetone-d $), 1.72\left(3 \mathrm{H}, \mathrm{s}, \mathrm{CH}_{3}, \mathrm{C} 7\right), 1.76(3 \mathrm{H}, \mathrm{s}), 2.70-2.80(1$ $\mathrm{H}, \mathrm{m}), 2.88-2.96(1 \mathrm{H}, \mathrm{m}), 3.65-3.74(1 \mathrm{H}, \mathrm{m}), 4.19-4.23(1 \mathrm{H}, \mathrm{m}), 4.54(1 \mathrm{H}, \mathrm{d}, J 13.6 \mathrm{~Hz}), 4.61-4.70(1 \mathrm{H}$, m), $4.82(1 \mathrm{H}, \mathrm{dd}, J$ 13.6, $2.8 \mathrm{~Hz}), 6.11(1 \mathrm{H}, \mathrm{d}, J 2.4 \mathrm{~Hz}), 6.80(4 \mathrm{H}, \mathrm{t}, J 6.8 \mathrm{~Hz}), 6.94(8 \mathrm{H}, \mathrm{t}, J 7.2 \mathrm{~Hz})$, $7.36(8 \mathrm{H}, \mathrm{m}), 7.51(1 \mathrm{H}, \mathrm{t}, J 7.6 \mathrm{~Hz}), 7.59-7.88(3 \mathrm{H}, \mathrm{m}), 7.85(2 \mathrm{H}, \mathrm{d}, J 8.4 \mathrm{~Hz}), 7.95(2 \mathrm{H}, \mathrm{d}, J 8.8 \mathrm{~Hz})$, 
$9.28(1 \mathrm{H}, \mathrm{s}) ; \delta_{\mathrm{C}}\left(100 \mathrm{MHz}\right.$; acetone- $\left.\mathrm{d}_{6}\right), 19.2,25.9,30.0,52.8,63.4,66.4,71.9,102.2,122.7,125.3,125.8$, 126.4 , 128.3, 129.7, 129.8, 135.9, 137.4, 138.4, 140.1, 145.0, 149.0, 165.0, 169.5; m/z 367.1658; $\mathrm{C}_{21} \mathrm{H}_{23} \mathrm{~N}_{2} \mathrm{O}_{4}$ (cation) requires 367.1658 .

(+)- $N$-((4S,5S)-2,2-Dimethyl-4-(4-(methylthio)phenyl)-1,3-dioxan-5-yl)-3,4-dihydroisoquinolinium tetraphenylborate $(3) .^{5}$

Prepared according to the general procedure from (4S,5S)-5-amino-2,2-dimethyl-4-(4-(methylthio)phenyl)1,3-dioxane $(0.50 \mathrm{~g}, 2.0 \mathrm{mmol})$, and purified by recrystallization from $\mathrm{CH}_{2} \mathrm{Cl}_{2} /$ hexane to give 3 as yellow plates (1.00 g, 73\%); m.p. 146-148 ${ }^{\circ} \mathrm{C}(\mathrm{dec}.) ;[\alpha]^{20}{ }_{\mathrm{D}}+115.9^{\circ}$ (c 1.41, acetone); Found: C, 79.05; H, 6.59; N, 1.93. $\mathrm{C}_{46} \mathrm{H}_{46} \mathrm{BNO}_{2} \mathrm{~S} \cdot 0.5 \mathrm{H}_{2} \mathrm{O}$ requires $\mathrm{C}, 79.27 ; \mathrm{H}, 6.66 ; \mathrm{N}, 2.01$; $v_{\max }\left(\right.$ film) $/ \mathrm{cm}^{-1} 3053,2996,2360,2341$, $1634,1603,1571,1478,1265,1201,1162,1108,1075 ; \delta_{\mathrm{H}}\left(250 \mathrm{MHz}\right.$; acetone-d $\left.\mathrm{d}_{6}\right) 1.66(3 \mathrm{H}, \mathrm{s}), 1.72(3 \mathrm{H}, \mathrm{s})$, $2.42(3 \mathrm{H}, \mathrm{s}), 2.66-2.84(1 \mathrm{H}, \mathrm{m}), 2.90-3.03(1 \mathrm{H}, \mathrm{m}), 3.62-3.74(1 \mathrm{H}, \mathrm{m}), 4.13-4.26(1 \mathrm{H}, \mathrm{m}), 4.50-4.55(2 \mathrm{H}$, m), $4.78(1 \mathrm{H}, \mathrm{dd}, J 13.8,3.1 \mathrm{~Hz}), 5.91(1 \mathrm{H}, \mathrm{d}, J 2.6 \mathrm{~Hz}), 6.78(4 \mathrm{H}, \mathrm{t}, J 7.2 \mathrm{~Hz}), 6.92(8 \mathrm{H}, \mathrm{t}, J$ 7.40 Hz), $\left.7.34(8 \mathrm{H}, \mathrm{m}), 7.40-7.56(6 \mathrm{H}, \mathrm{m}), 7.76-7.87(2 \mathrm{H}, \mathrm{m}), 9.30(1 \mathrm{H}, \mathrm{s}) ; \delta_{\mathrm{C}}(100 \mathrm{MHz} \text {; acetone-d })_{6}\right) 15.4,18.8$, 25.4, 30.0, 52.3, 62.7, 66.5, 71.5, 101.4, 122.3, 125.3, 126.1, 126.9, 127.4, 129.2, 129.3, 133.9, 135.2, 137.0, $137.8,139.5,140.5,165.0,168.5 ; \mathrm{m} / z$ 368.1682; $\mathrm{C}_{22} \mathrm{H}_{26} \mathrm{NO}_{2} \mathrm{~S}$ (cation) requires 368.1684.

\section{(-)- $N$-((4R,5R)-2,2-Dimethyl-4-[4-methoxyphenyl)-1,3-dioxan-5-yl)-3,4-dihydroisoquinolinium}

\section{tetraphenylborate (4). ${ }^{5}$}

Prepared according to the general procedure from (-)-(4R,5R)-5-amino-2,2-dimethyl-4-(4-methoxyphenyl)1,3-dioxane $(0.40 \mathrm{~g}, 1.7 \mathrm{mmol})$, and purified by recrystallization from $\mathrm{CH}_{2} \mathrm{Cl}_{2} /$ hexane to give 4 as yellow plates $(0.83 \mathrm{~g}, 74 \%)$; m.p. $171-173{ }^{\circ} \mathrm{C}$ (dec.); $[\alpha]^{20}{ }_{\mathrm{D}}-108.6^{\circ}$ (c 1.40, acetone); $v_{\max }($ film $) / \mathrm{cm}^{-1} 1639,1604$, 1573, 1514, 1382, 1254, 1202, 1107, 1031; Found C, 77.70; H, 6.50; N, 1.88. $\mathrm{C}_{46} \mathrm{H}_{46} \mathrm{BNO}_{3} \cdot 0.5 \mathrm{Et}_{2} \mathrm{O}$ requires C, 77.92; H, 6.54; N, 1.98.; $\delta_{\mathrm{H}}\left(400 \mathrm{MHz} ; \mathrm{CDCl}_{3}\right) 1.51(3 \mathrm{H}, \mathrm{s}), 1.52(3 \mathrm{H}, \mathrm{s}), 2.20-2.25(1 \mathrm{H}, \mathrm{m}), 2.31-2.35$ (1 H, m), 2.85-2.90 (1 H, m), 3.00-3.10 (1 H, m), 2.96-3.07 (1 H, m), $3.56(1 \mathrm{H}, \mathrm{d}, J 14.4 \mathrm{~Hz}), 3.72(3 \mathrm{H}, \mathrm{s})$, $3.90(1 \mathrm{H}, \mathrm{dd}, J$ 14.0, $2.8 \mathrm{~Hz}), 5.11(1 \mathrm{H}, \mathrm{d}, J 2.4 \mathrm{~Hz}), 6.79(2 \mathrm{H}, \mathrm{d}, J 2.0 \mathrm{~Hz}), 6.87(4 \mathrm{H}, \mathrm{t}, J 7.2 \mathrm{~Hz}), 7.02(8$ H, t, J 7.6 Hz), 7.23-7.24 (2 H, m) 7.24-7.31 (1 H, m), 7.41-7.57 (8 H, m), 7.60-7.69 (1 H, m), $8.25(1 \mathrm{H}, \mathrm{s})$; $\delta_{\mathrm{C}}\left(100 \mathrm{MHz} ; \mathrm{CDCl}_{3}\right) 18.4,24.6,29.4,50.5,55.4,61.9,64.9,70.5,100.4,114.4,122.2,123.7,125.9,127.3$, $127.9,128.7,129.5,134.0,134.7,136.2,138.8,159.8,163.8,169.5 ; \mathrm{m} / \mathrm{z} 352.1915 ; \mathrm{C}_{22} \mathrm{H}_{26} \mathrm{NO}_{3}$ (cation) requires 352.1913 .

(+)-N-((4S,5S)-2,2-Dimethyl-4-(4-(methylsulfonyl)phenyl)-1,3-dioxan-5-yl)-3,4-dihydroisoquinolinium tetraphenylborate $(6){ }^{6}$

Prepared according to the general procedure from (4S,5S)-5-amino-2,2-dimethyl-4-[4(methylsulfonyl)phenyl]-1,3-dioxane $(0.75 \mathrm{~g}, 2.96 \mathrm{mmol})$, and purified by recrystallization from $\mathrm{CH}_{2} \mathrm{Cl}_{2} /$ hexane to give 6 as yellow plates (1.55 g, 73\%); m.p. 199-201 ${ }^{\circ} \mathrm{C}\left(\mathrm{dec}\right.$.); $[\alpha]^{20}{ }_{\mathrm{D}}+126.7^{\circ}(c) 1.20$, acetone); Found: C, 75.62; H, 6.32; N, 1.84. $\mathrm{C}_{46} \mathrm{H}_{46} \mathrm{BNO}_{4} \mathrm{~S} \cdot 0.5 \mathrm{H}_{2} \mathrm{O}$ requires $\mathrm{C}, 75.79 ; \mathrm{H}, 6.50 ; \mathrm{N}, 1.92$.; $v_{\max }($ film $) / \mathrm{cm}^{-1} 1636,1603,1572,1478,1383,1314,1266,1202,1150,1076,1032,956 ; \delta_{\mathrm{H}}(400 \mathrm{MHz}$; acetone- $\left._{6}\right), 1.69(3 \mathrm{H}, \mathrm{s}), 1.72(3 \mathrm{H}, \mathrm{s}), 2.60-2.69(1 \mathrm{H}, \mathrm{m}), 2.85-2.96(1 \mathrm{H}, \mathrm{m}), 3.00(3 \mathrm{H}, \mathrm{s}), 3.65-3.72(1 \mathrm{H}$, m), 4.12-4.20 (1 H, m), 4.49 (1 H, d, J 13.6 Hz), 4.50-4.64 (1 H, m), 4.77 (1 H, dd, J 13.6, $2.8 \mathrm{~Hz}), 6.05$ (1 
H, d, J 2.8 Hz), $6.80(4 \mathrm{H}, \mathrm{t}, J 7.2 \mathrm{~Hz}), 6.92(8 \mathrm{H}, \mathrm{t}, J 7.2 \mathrm{~Hz}), 7.31-7.44(8 \mathrm{H}, \mathrm{m}), 7.49(1 \mathrm{H}, \mathrm{t}, J$ 7.6 Hz), 7.73-7.83 (3 H, m), $7.82(2 \mathrm{H}, \mathrm{d}, J 8.2 \mathrm{~Hz}), 7.95(2 \mathrm{H}, \mathrm{d}, J 8.2 \mathrm{~Hz}), 9.28(1 \mathrm{H}, \mathrm{s}) ; \delta_{\mathrm{C}}(100 \mathrm{MHz} \text {; acetone-d })_{6}$, 18.8, 25.4, 29.5, 44.3, 52.3 62.9, 66.1, 71.5, 101.7, 122.3, 125.3, 126.1, 127.6, 128.8, 129.3, 129.4, 135.4, 137.0, 137.0, 137.9, 142.4, 143.2, 165.0, 168.9; $m / z$ 400.1586; $\mathrm{C}_{22} \mathrm{H}_{26} \mathrm{NO}_{4} \mathrm{~S}$ (cation) requires 400.1583.

\section{Tetraphenylphosphonium monoperoxysulfate.}

Oxone ${ }^{\mathrm{TM}}$ triple salt $\left(2 \mathrm{KHSO}_{5}: \mathrm{KHSO}_{4}: \mathrm{K}_{2} \mathrm{SO}_{4}\right)\left(15.0 \mathrm{~g}, 48.8 \mathrm{mmol}\right.$ with respect to $\left.\mathrm{KHSO}_{5}\right)$ was dissolved in deionized water $(300 \mathrm{ml})$, and the solution stirred at $10-15{ }^{\circ} \mathrm{C}$ (water bath). A solution of tetraphenylphosphonium chloride $(15.0 \mathrm{~g}, 40.0 \mathrm{mmol})$ in distilled dichloromethane $(300 \mathrm{ml})$ was added over $5 \mathrm{~min}$, and the mixture stirred for an additional $30 \mathrm{~min}$. The organic layer was separated, and the solvent removed under reduced pressure at room temperature. The colourless residue, the crude salt, was transferred to a fritted glass funnel and washed with distilled water $(2 \times 75 \mathrm{ml})$. The solid was dissolved in dichloromethane $(180 \mathrm{ml})$, and the solution dried $\left(\mathrm{MgSO}_{4}\right)$. Hexane was added until cloudiness developed, and the flask was placed in the freezer $\left(-20^{\circ} \mathrm{C}\right)$ overnight, producing a colourless precipitate of the salt about $85 \%$ pure in peroxide $(15.4 \mathrm{~g}, 70 \%) . \delta \mathrm{H}\left(250 \mathrm{MHz} ; \mathrm{CDCl}_{3}\right) 7.62-7.65(8 \mathrm{H}, \mathrm{m}), 7.76-7.81(8 \mathrm{H}, \mathrm{m})$, 7.88-7.92 (4 H, m), $8.92(1 \mathrm{H}, \mathrm{s})$.

General procedure for catalytic asymmetric epoxidation of simple alkenes mediated by iminium salts using tetraphenylphosphonium monoperoxysulfate.

Tetraphenylphosphonium monoperoxysulfate ( 2 equiv. with respect to the alkene) was dissolved in the desired solvent ( $2 \mathrm{ml}$ per $0.1 \mathrm{~g}$ oxidant) and the solution cooled to the required temperature. To this was added the iminium salt as a solution in the solvent $(0.5 \mathrm{ml}$ per $0.1 \mathrm{~g}$ oxidant $)$. This iminium salt solution was cooled to the same temperature as the solution containing the oxidant and added to it dropwise over 15-20 min; the temperature of the reaction vessel was monitored to minimize increase in temperature during the addition. A solution of the alkene in the reaction solvent $(0.5 \mathrm{ml}$ per $0.1 \mathrm{~g}$ oxidant $)$ was added dropwise. The mixture was stirred at the reaction temperature until the alkene was completely consumed according to tlc. Diethyl ether (pre-cooled to the reaction temperature) $(20 \mathrm{ml}$ per $0.1 \mathrm{~g}$ oxidant) was added to induce precipitation of the remaining oxidant, and the mixture filtered through Celite. The solvents were removed, diethyl ether $(40 \mathrm{ml})$ was added to the residue, and the solution passed through a short pad of silica gel to remove catalyst residues. The solvents were removed to give the epoxide. If the reaction does not reach completion the epoxide can be separated from the alkene by column chromatography, eluting with ethyl acetate/light petroleum 1:99.

\section{General procedure for the catalytic asymmetric epoxidation of alkenes mediated by iminium salts using Oxone.}

Oxone ( 2 equiv. with respect to alkene) was added with stirring to an ice-cooled solution of sodium carbonate (4 equiv.) in water ( $12 \mathrm{ml}$ per $1.50 \mathrm{~g}$ of sodium carbonate), and the resulting foaming solution stirred for 5-10 minutes, until most of the initial effervescence subsided. A solution of the iminium salt (10 mol\% with respect to alkene) in acetonitrile $(6 \mathrm{ml}$ per $1.50 \mathrm{~g}$ of sodium carbonate used) was added, followed by a solution of the alkene in acetonitrile $(6 \mathrm{ml}$ per $1.50 \mathrm{~g}$ of sodium carbonate used). The suspension was 
stirred with ice bath cooling until the substrate was completely consumed according to tlc. The reaction mixture was diluted with ice-cooled diethyl ether $(20 \mathrm{ml}$ per $100 \mathrm{mg}$ substrate) and the same volume of water added immediately. The aqueous phase was washed four times with diethyl ether, and the combined organic solutions washed with brine and dried $\left(\mathrm{MgSO}_{4}\right)$. Filtration and removal of the solvents gave a yellow or light brown residue, which was purified by column chromatography, typically using ethyl acetate/light petroleum (1:99), to provide the pure epoxide.

\section{General procedure for the formation of racemic epoxides.}

The alkene was dissolved in $\mathrm{CH}_{2} \mathrm{Cl}_{2}(10 \mathrm{ml} / \mathrm{g})$ and the solution cooled using an ice bath. A solution of $\mathrm{m}$ CPBA (2 eq.) in $\mathrm{CH}_{2} \mathrm{Cl}_{2}\left(10 \mathrm{ml} / \mathrm{g}\right.$, pre-dried over $\left.\mathrm{MgSO}_{4}\right)$ was added. The reaction was allowed to reach ambient temperature and stirred until complete consumption of the substrate was observed by tlc. Saturated aqueous $\mathrm{NaHCO}_{3}(10 \mathrm{ml} / \mathrm{g})$ was added and the layers separated. The organic layer was washed with saturated aqueous $\mathrm{NaOH}(1.0 \mathrm{M}, 10 \mathrm{ml} / \mathrm{g})$ and dried $\left(\mathrm{MgSO}_{4}\right)$. The solvents were removed under reduced pressure, and the residue purified by column chromatography, typically eluting with ethyl acetate/light petroleum (1:99), to give the pure epoxide.

trans- $\alpha$-Methylstilbene oxide. ${ }^{10}$ Colourless oil; $v_{\max }($ neat $) / \mathrm{cm}^{-1} 3061,1602,1495,1449,1381,1279,1157$, 1118, 1065, 1027, 980; $\delta_{\mathrm{H}}\left(400 \mathrm{MHz} ; \mathrm{CDCl}_{3}\right) 1.46(3 \mathrm{H}, \mathrm{s}), 3.96(1 \mathrm{H}, \mathrm{s}), 7.30-7.46(10 \mathrm{H}, \mathrm{m}) ; \delta_{\mathrm{C}}(100 \mathrm{MHz}$; $\left.\mathrm{CDCl}_{3}\right) 17.1,63.5,67.5,125.6,126.9,127.7,127.9,128.6,129.2,136.4,142.8$.

Triphenylethylene oxide. ${ }^{11}$ Colourless oil which slowly solidified, mp 66-67 ${ }^{\circ} \mathrm{C}$, (lit. mp $75{ }^{\circ} \mathrm{C}$ ); $v_{\max }($ neat $) / \mathrm{cm}^{-1} 3062,3030,2957,2925,2856,1605,1596,1499,1471,1448,1262,1221,741,698,621$; $\delta_{\mathrm{H}}\left(250 \mathrm{MHz} ; \mathrm{CDCl}_{3}\right)$ 4.39-4.41 $(1 \mathrm{H}, \mathrm{m}), 7.10-7.47(15 \mathrm{H}, \mathrm{m}) ; \delta_{\mathrm{C}}\left(62.5 \mathrm{MHz} ; \mathrm{CDCl}_{3}\right)$ 68.0, 68.3, 126.3, 126.8, 127.5, 127.6, 127.7, 127.8 128.0, 128.2, 128.6, 135.4, 135.9, 141.1.

1-Phenylcyclohexene oxide. ${ }^{12}$ Colourless oil; $v_{\max }\left(\right.$ neat) $/ \mathrm{cm}^{-1}$ 3084, 1602, 1495, 1446, 1359, 1249, 1173 , 1132 , 1079, 1030, 993, 974; $\delta_{\mathrm{H}}\left(250 \mathrm{MHz} ; \mathrm{CDCl}_{3}\right) 1.22-1.35(1 \mathrm{H}, \mathrm{m}), 1.53-1.64(3 \mathrm{H} \mathrm{m}), 1.99-2.06(2 \mathrm{H}, \mathrm{m})$ 2.16-2.18 (1 H, m), 2.26-2.32 (1 H, m), $3.10(1 \mathrm{H}, \mathrm{t}, J 2.0 \mathrm{~Hz}), 7.28-7.44(5 \mathrm{H}, \mathrm{m}) ; \delta_{\mathrm{C}}\left(62.5 \mathrm{MHz} ; \mathrm{CDCl}_{3}\right)$ $19.8,20.1,24.7,28.2,60.1,61.8,125.3,127.1,128.2,142.8$.

1-Phenyl-3,4-dihydronaphthalene oxide. ${ }^{12}$ Pale yellow solid; mp 104-106 ${ }^{\circ} \mathrm{C}$, (lit. ${ }^{13} \mathrm{mp} 94-97{ }^{\circ} \mathrm{C}$ ); $v_{\max }($ nujol $) / \mathrm{cm}^{-1} 1602,1486,1307,1155,1074,1042,953 ; \delta_{\mathrm{H}}\left(250 \mathrm{MHz} ; \mathrm{CDCl}_{3}\right) 2.10(1 \mathrm{H}, \mathrm{td}, J 5.8,13.7$ Hz), 2.49-2.60 (1 H, m), 2.77 (1 H, dd, J 5.6, 15.5 Hz), 2.98-3.06 (1 H, m), 3.71 (1 H, d, J 3.1 Hz), 7.11-7.31 (4 H, m), 7.45-7.61 (5 H, m); $\delta_{\mathrm{C}}\left(62.5 \mathrm{MHz} ; \mathrm{CDCl}_{3}\right)$ 22.1, 25.4, 60.9, 63.0, 126.0, 127.7, 127.9, 128.1, 128.2, 128.6, 129.8, 135.0, 137.5, 138.8 .

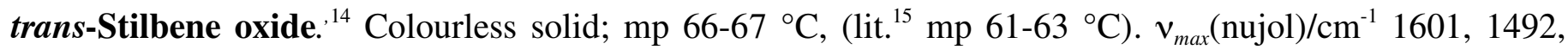
1284, 1176, 1157, 1094, 1072, 1025; $\delta_{\mathrm{H}}\left(400 \mathrm{MHz} ; \mathrm{CDCl}_{3}\right) 3.84(2 \mathrm{H}, \mathrm{s}), 7.28-7.37(10 \mathrm{H} \mathrm{m}) ; \delta_{\mathrm{C}}(100 \mathrm{MHz}$; $\left.\mathrm{CDCl}_{3}\right) 63.3,126.0,128.6,129.3,137.6$. 
Indene oxide: ${ }^{16}$ Colourless oil; $v_{\max }($ neat $) / \mathrm{cm}^{-1} 3027,2917,1482,1464,1390,1372,1232,1183,1142,829$, 758, 745, 723; $\delta_{\mathrm{H}}\left(250 \mathrm{MHz} ; \mathrm{CDCl}_{3}\right) 2.97(1 \mathrm{H}, \mathrm{dd}, J 2.7$ and $18.1 \mathrm{~Hz}), 3.21(1 \mathrm{H}, \mathrm{d}, J 17.6 \mathrm{~Hz}), 4.13(1 \mathrm{H}, \mathrm{t}$, $J 3.0 \mathrm{~Hz}), 4.26(1 \mathrm{H}, \mathrm{dd}, J 1.1$ and $2.8 \mathrm{~Hz}), 7.14-7.29(3 \mathrm{H}, \mathrm{m}), 7.49(1 \mathrm{H}, \mathrm{dd}, J 1.7$ and $6.6 \mathrm{~Hz}) ; \delta_{\mathrm{C}}(100$ $\left.\mathrm{MHz} ; \mathrm{CDCl}_{3}\right)$ 34.6, 57.6, 59.1, 125.2, 126.1, 126.3, 128.6, 141.0, 143.6.

1,2-Dihydronapthylene oxide: ${ }^{17}$ Colourless oil; $v_{\max }\left(\right.$ neat) $/ \mathrm{cm}^{-1}$ 3059, 3028, 2930, 2850, 1602, 1493, 1316, 1129, 1088, 1030, 964; $\delta_{\mathrm{H}}\left(400 \mathrm{MHz} ; \mathrm{CDCl}_{3}\right)$ 1.60-1.70 (1 H, m), 2.31-2.39 (1 H, m, ), 2.45 (1 H, dd, J 15.6 and 5.6 Hz), 2.65-2.70 (1 H, m), $3.65(1 \mathrm{H}, \mathrm{t}, J 4.0 \mathrm{~Hz}), 3.78(1 \mathrm{H}, \mathrm{d}, J 4.4 \mathrm{~Hz}), 7.01(1 \mathrm{H}, \mathrm{d}, J$ 7.2 Hz), 7.14$7.23(2 \mathrm{H}, \mathrm{m}), 7.33(1 \mathrm{H}, \mathrm{d}, J 7.2 \mathrm{~Hz}) ; \delta_{\mathrm{C}}\left(100 \mathrm{MHz} ; \mathrm{CDCl}_{3}\right) 22.2$ 24.8, 55.2, 55.5, 126.5, 128.8, 128.8, $129.9,132.9,137.1$.

cis- $\beta$-Methylstyrene oxide: ${ }^{17}$ Colourless oil; $\boldsymbol{v}_{\max }($ neat $) / \mathrm{cm}^{-1} 3061,2994,1604,1496,1450,1258,1149$, 9563, 853, 742, 700, 619; $\delta_{\mathrm{H}}\left(250 \mathrm{MHz} ; \mathrm{CDCl}_{3}\right)$ 1.12, (3 H, d, J 5.4 Hz), 3.32-3.40 (1 H, m), $4.08(1 \mathrm{H}, \mathrm{d}, J$ $4.3 \mathrm{~Hz}), 7.24-7.39(5 \mathrm{H}, \mathrm{m}) ; \delta_{\mathrm{C}}\left(62.5 \mathrm{MHz} ; \mathrm{CDCl}_{3}\right) 12.8,55.4,57.8,126.9,127.7,128.3,135.8$.

cis-2,3-Epoxyheptene: ${ }^{18}$ Colourless oil, 68\% yield; $v_{\max }$ (neat) $/ \mathrm{cm}^{-1}$ 2959, $29302874,1390,1259,1218$, $1151,1114,1032,752 ; \delta_{\mathrm{H}}\left(400 \mathrm{MHz} ; \mathrm{CDCl}_{3}\right) 0.93(3 \mathrm{H}, \mathrm{t}, J 8.0 \mathrm{~Hz}), 1.20(3 \mathrm{H}, \mathrm{d}, J 4.0 \mathrm{~Hz}), 1.32-1.50(6 \mathrm{H}$, m), 2.86-2.91 (1 H, m), 2.99-3.06 (1 H, m); $\delta_{\mathrm{C}}\left(100 \mathrm{MHz} ; \mathrm{CDCl}_{3}\right)$ 12.2, 13.0, 21.6, 26.2, 27.6, 51.6, 56.1.

6-Cyanobenzopyran oxide: ${ }^{19}$ Colourless oil which solidified: $v_{\max }\left(\right.$ film) $/ \mathrm{cm}^{-1} 3089,3038,2979,2934,2226$, 1615, 1579, 1490, 1346, 1279, 1157, 1107, 1046, 955; $\delta_{\mathrm{H}}\left(400 \mathrm{MHz} ; \mathrm{CDCl}_{3}\right) 1.22(3 \mathrm{H}, \mathrm{s}), 1.52(3 \mathrm{H}, \mathrm{s})$, $3.47(1 \mathrm{H}, \mathrm{d}, J 4.4 \mathrm{~Hz}), 3.86(1 \mathrm{H}, \mathrm{d}, J 4.4 \mathrm{~Hz}), 6.79(1 \mathrm{H}, \mathrm{d}, J 8.4 \mathrm{~Hz}), 7.45(1 \mathrm{H}, \mathrm{dd}, J$ 2.0, $8.4 \mathrm{~Hz}), 7.58(1$ $\mathrm{H}, \mathrm{d}, J 2.4 \mathrm{~Hz}) ; \delta_{\mathrm{C}}\left(100 \mathrm{MHz} ; \mathrm{CDCl}_{3}\right)$ 23.4, 25.9, 50.3, 62.7, 75.1, 104.7, 119.0, 119.2, 121.5, 134.2, 134.8, 156.87 .

6-Nitrobenzopyran oxide: ${ }^{19}$ Colourless oil which solidified: $v_{\max }\left(\right.$ film) $/ \mathrm{cm}^{-1} 3075,2926,2850,1621,1590$, 1518, 1344, 1281, 1209, 1160, 1088, 955; $\delta_{\mathrm{H}}\left(400 \mathrm{MHz} ; \mathrm{CDCl}_{3}\right) 1.33(3 \mathrm{H}, \mathrm{s}), 1.63(3 \mathrm{H}, \mathrm{s}), 3.57$ (1 H, dd, $J$ $4.4 \mathrm{~Hz}), 4.00(1 \mathrm{H}, \mathrm{d}, J 4.4 \mathrm{~Hz}), 6.89(1 \mathrm{H}, \mathrm{d}, J 9.0 \mathrm{~Hz}), 8.15(1 \mathrm{H}, \mathrm{dd}, J 2.8,9.0 \mathrm{~Hz}), 8.30(1 \mathrm{H}, \mathrm{d}, J 2.8 \mathrm{~Hz})$; $\delta_{\mathrm{C}}\left(100 \mathrm{MHz} ; \mathrm{CDCl}_{3}\right) 23.1,25.5,50.0,62.1,75.2,118.5,120.3,125.8,126.3,141.5,158.3$.

6-Chlorobenzopyran oxide: ${ }^{19}$ Colourless oil: $v_{\max }\left(\right.$ film) $/ \mathrm{cm}^{-1} 3080,2985,2933,1611,1579,1478,1366$, 1339, 1268, 1237, 1202, 1168, 1103, 1087, 1036, 954; $\delta_{\mathrm{H}}\left(400 \mathrm{MHz} ; \mathrm{CDCl}_{3}\right) 1.17(3 \mathrm{H}, \mathrm{s}), 1.50(3 \mathrm{H}, \mathrm{s})$, $3.41(1 \mathrm{H}, \mathrm{dd}, J 4.4 \mathrm{~Hz}), 3.77(1 \mathrm{H}, \mathrm{d}, J 4.4 \mathrm{~Hz}), 6.67(1 \mathrm{H}, \mathrm{d}, J 8.6 \mathrm{~Hz}), 7.11(1 \mathrm{H}, \mathrm{dd}, J 2.6$ and $8.6 \mathrm{~Hz})$, $7.24(1 \mathrm{H}, \mathrm{d}, J 2.6 \mathrm{~Hz}) ; \delta_{\mathrm{C}}\left(100 \mathrm{MHz} ; \mathrm{CDCl}_{3}\right)$ 22.5, 25.6, 50.4, 62.6, 73.4, 119.4, 121.6, 125.7, 129.2, 130.2, 151.2.

1 Avecia LifeScience Molecules, P.O. Box 521, Leeds Road, Huddersfield, HD2 1GA, England.

2 Picot, A.; Millet, P.; Lusinchi, X. Tetrahedron Lett., 1976, 17, 1573; Hanquet, G.; Lusinchi, X.; Milliet, P. Tetrahedron Lett., 1987, 28, 6061; Hanquet, G.; Lusinchi, X.; Milliet, P. Tetrahedron Lett., 1988, 29, 3941. 
Bohé, L.; Hanquet, G.; Lusinchi, M.; Lusinchi, X. Tetrahedron Lett., 1993, 34, 7271; Bohé, L.; Lusinchi, M.; Lusinchi, X. Tetrahedron, 1999, 55, 141; Bohé, L.; Kammoun, M. Tetrahedron Lett., 2002, 43, 803; Bohé, L.; Kammoun, M. Tetrahedron Lett., 2004, 45, 747; Gluszynska, A.; Mackowska, I.; Rozwadowska, M. D.; Sienniak, W. Tetrahedron: Asymmetry 2004, 15, 2499. Aggarwal, V. K.; Wang, M. F. J. Chem. Soc., Chem. Commun., 1996, 191; Armstrong, A.; Ahmed, G.; Garnett, I.; Gioacolou, K. Synlett, 1997, 1075; Armstrong, A.; Ahmed, G.; Garnett, I.; Gioacolou, K.; Wailes, J. S. Tetrahedron, 1999, 55, 2341; Minakata, S.; Takemiya, A.; Nakamura, K.; Ryu, I.; Komatsu, M. Synlett, 2000, 12, 1810. Wong, M-K.; Ho, L-M.; Zheng, Y-S.; Ho, C-Y.; Yang, D. Org. Lett., 2001, 16, 2587. Lacour, J.; Monchaud, D.; Marsol, C. Tetrahedron Lett., 2002, 43, 8257;

Vachon, J.; Pérollier, C.; Monchaud, D.; Marsol, C.; Ditrich, K.; Lacour, J J. Org. Chem. 2005, 70, 5903.

Page, P. C. B.; Rassias, G. A.; Bethell, D.; Schilling, M. B. J. Chem. Soc., Perkin Trans., 1, 2000, 3325.

Page, P. C. B.; Rassias, G. A.; Barros, D.; Ardakani, A.; Buckley, B.; Bethell, D.; Smith, T. A. D.; Slawin, A. M. Z. J. Org. Chem., 2001, 66, 6926; Page, P. C. B.; Rassias, G. A.; Barros, D.; Ardakani, A.; Bethell, D.; Merrifield, E. Synlett, 2002, 4, 580; Page, P. C. B.; Buckley, B. R.; Rassias, G. A.; Blacker, A. J. J. Org. Chem. 2005, Submitted.

Page, P. C. B.; Buckley, B. R.; Heaney, H.; Blacker, A. J. Org. Lett. 2005, 7, 375.

Page, P. C. B.; Buckley, B. R.; Blacker, A. J. Org. Lett. 2004, 6, 1543.

Page, P. C. B.; Barros, D.; Buckley, B. R.; Ardakani, A.; Marples, B. A. J. Org. Chem. 2004, 69. 3595.

9 Campestrini, S.; Di Furia, G.; Labat, G.; Novello, F. J. Chem. Soc., Perkin Trans., 1, 1994, 2175.

10 Sasaki, H.; Irie, R.; Hamada, T.; Suzuki, K.; Katsuki, T. Tetrahedron, 1994, 50, 11827; Boyd, D. R.; Sharma, N. D.; Bowers, N. I.; Goodrich, P. A.; Groocok, R. M. Tetrahedron: Asymmetry, 1996, 7, 1559; Sola, L.; Vidal-Ferran, A.; Moyano, A.; Pericas, M. A.; Riera, A. Tetrahedron: Asymmetry, 1997, 8, 1559.

11 Fleiser, R.; Galle, D.; Braun, M. Liebigs Ann., 1997, 6, 1189.

12 Tu, Y.; Wang, Z.-X.; Shi, Y. J. Am. Chem. Soc., 1996, 118, 9806; Brandes, B. D.; Jacobsen, E. N. J. Org. Chem., 1994, 59, 4378; Belluci, G. J. Chem. Soc., Perkin Trans. 2, 1973, 292.

13 Padwa, A.; Owens, D. J. Org. Chem., 1977, 42, 3076.

14 Tian, H.; She, X.; Yu, H.; Shu, L.; Shi, Y. J. Org. Chem. 2002, 67, 2435.

15 Solladié-Cavallo, A.; Diep-Vohuule, A.; Sunjic, V.; Vinkovic, V. Tetrahedron: Asymmetry, 1996, 7, 1783; Chang, H.-T.; Sharpless, K. B. J. Org. Chem., 1996, 61, 6456.

16 Sasaki, H.; Irie, R.; Hamada, T.; Suzuki, K.; katsuki, T. Tetrahedron, 1994, 50, 11827; Boyd, D. R.; Sharma, N. D.; Bowers, N. I.; Goodrich, P. A.; Groocok, R. M. Tetrahedron: Asymmetry, 1996, 7, 1559; Sola, L.; Vidal-Ferran, A.; Moyano, A.; Pericas, M. A.; Riera, A. Tetrahedron: Asymmetry, 1997, 8, 1559.

17 Tian, H.; She, X.; Yu, H.; Shu, L.; Shi, Y. J. Org. Chem. 2002, 67, 2435.

18 Kurth, M. J.; Abreo, M. A. Tetrahedron, 1990, 46, (15), 5085; b) Krief, A.; Heresi, L.; Nagy, J. B.; Derouane, E. G. Angew. Chem., 1977, 89, 103; Chiappe, C.; Cordoni, A.; Lo Moro, G.; Palese, C. D. Tetrahedron: Asymmetry, 1998, 9, 341. 
19 Evans, J. M.; Fake, C. S.; Hamilton, T. C.; Poyser, R. H.; Watts, E. A. J. Med. Chem. 1983, 26, 1582; Bergmann, R.; Eiermann, V.; Gericke, R. J. Med. Chem. 1990, 33, 2759; Lee, N. H.; Muci, A. R.; Jacobsen, E. N. Tetrahedron Lett. 1991, 32, 5055. 